\title{
Functional Imaging Biomarkers Predicting Upper Limb Recovery from Severe Motor Impairment after Stroke
}

Jungsoo Lee ${ }^{1,2}$, Ahee Lee ${ }^{2}$, Heegoo Kim², Jinuk Kim², Kyung Ah Kim¹, Won Hyuk Chang'1, Yun-Hee Kim¹,2

${ }^{1}$ Department of Physical and Rehabilitation Medicine, Center for Prevention and Rehabilitation, Heart Vascular Stroke Institute, Samsung Medical Center, Sungkyunkwan University School of Medicine, ${ }^{2}$ Department of Health Sciences and Technology, Department of Medical Device Management \& Research, Department of Digital Health, Samsung Advanced Institute for Health Science and Technology, Sungkyunkwan University

\section{Introduction}

- Predicting recovery outcome after stroke are important to implicate individually-tailored rehabilitation strategy. In case of recovery of upper extremity (UE) motor function, most biomarkers demonstrated low predictive power in patients with severe motor impairment after stroke.

This study focused on investigation of neuroimaging biomarkers optimally predicting recovery of UE motor function in severe stroke patients. We hypothesized that there may be different primary neuroimaging markers depending on the pattern of later recovery.

\section{Methods}

\section{Participants \& Experimental Design}

- Forty-two subacute ischemic stroke patients with unilateral lesion

- Nineteen years or older at the time of stroke onset

- Fugl-Meyer assessment (FMA) score under 55 at 2 weeks after stroke

- All patients underwent T1-weighted, diffusion tensor imaging (DTI), resting-state functional MRI (rs-fMRI) data acquisition, as well as motor function assessment using the FMA at 2 weeks. FMA was assessed again at $3 \mathrm{~ms}$ after stroke.

- Based on proportional recovery rule, patients were divided into Fitter and Non-fitter groups according to their FMA-UE motor recovery at 3 ms whether the improvement achieve $70 \%$ of initial FMA score (Fitter) or not (Non-Fitter).

\begin{tabular}{|c|c|c|}
\hline Group & Fitters ( $n=23$ ) & Non-fitters $(n=19)$ \\
\hline \multicolumn{3}{|l|}{ Age (years) } \\
\hline Mean \pm SD & $52.3 \pm 11.2$ & $63.1 \pm 11.4$ \\
\hline \multicolumn{3}{|l|}{$\operatorname{Sex}(n)$} \\
\hline Male / Female & $15 / 8$ & $9 / 10$ \\
\hline \multicolumn{3}{|l|}{ Lesion side (n) } \\
\hline Right / Left & $9 / 14$ & $9 / 10$ \\
\hline \multicolumn{3}{|l|}{ Lesion location (n) } \\
\hline Supra- / Infra-tentorial & $14 / 9$ & $16 / 3$ \\
\hline \multicolumn{3}{|l|}{ Initial Impairment, mean \pm SD } \\
\hline FMA-UE & $18.0 \pm 9.7$ & $8.0 \pm 5.7$ \\
\hline NIHSS & $6.1 \pm 3.4$ & $12.7 \pm 5.4$ \\
\hline \multicolumn{3}{|l|}{ Time post stroke (days) } \\
\hline Mean \pm SD & $14.8 \pm 8.8$ & $17.0 \pm 7.5$ \\
\hline \multicolumn{3}{|l|}{ Risk factors (yes/no) } \\
\hline Hypertension & $14 / 9$ & $8 / 11$ \\
\hline Diabetes mellitus & $7 / 16$ & $4 / 15$ \\
\hline Hyperlipidemia & $9 / 14$ & $5 / 14$ \\
\hline Ischemic heart disease & $3 / 20$ & $0 / 19$ \\
\hline
\end{tabular}

\section{Data Processing \& Measurements}

\section{- Lesion load \& Lesion volume}

The individual lesion was manually drew and the CST template obtained from probabilistic tractography in the healthy DTI data. Lesion load was calculated by overlaying the individual lesion volume onto the CST template.

Lesion volume was calculated by counting the number of lesioned voxels from the lesion map and multiplying the voxel volume.

- Fractional anisotropy (FA) analysis from DTI

FSL software (eddy current correction, non-linearly registration)

Regional specific FA values using the Johns Hopkins University white matter atlas - posterior limb of the internal capsule (PLIC), cerebral peduncle $(\mathrm{CP})$, superior corona radiata $(\mathrm{SCR})$, superior cerebellar peduncle (SCP), genu of the corpus callosum (GCC), body of the corpus callosum (BCC), and splenium of the corpus callosum (SCC)

- Functional connectivity (FC) analysis from rs-fMRI

CONN toolbox (head motion, slice timing correction, scrubbing, spatial registration, segmentation, spatial smoothing, removal of nuisance sources, band-pass filtering, linear detrending)

- Automated anatomical labeling template-based functional connectivity

\section{Results}

\section{Group Lesion Map}

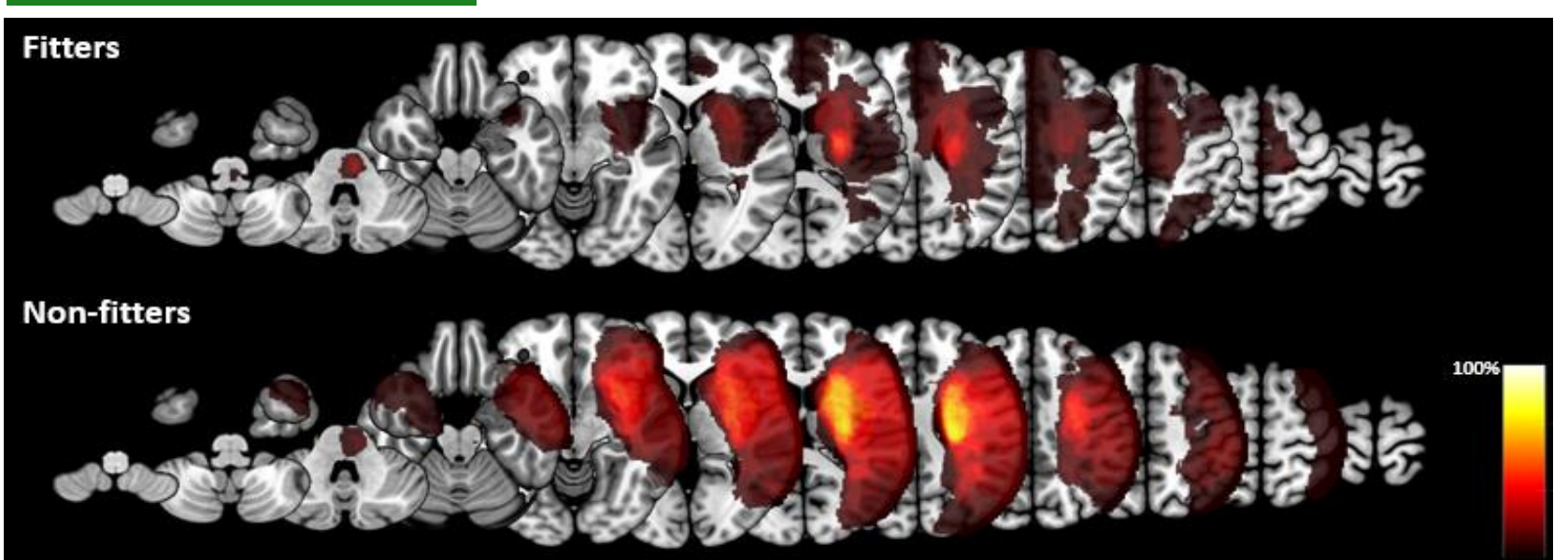

Functional Imaging Measurements: DTI, rs-fMRI
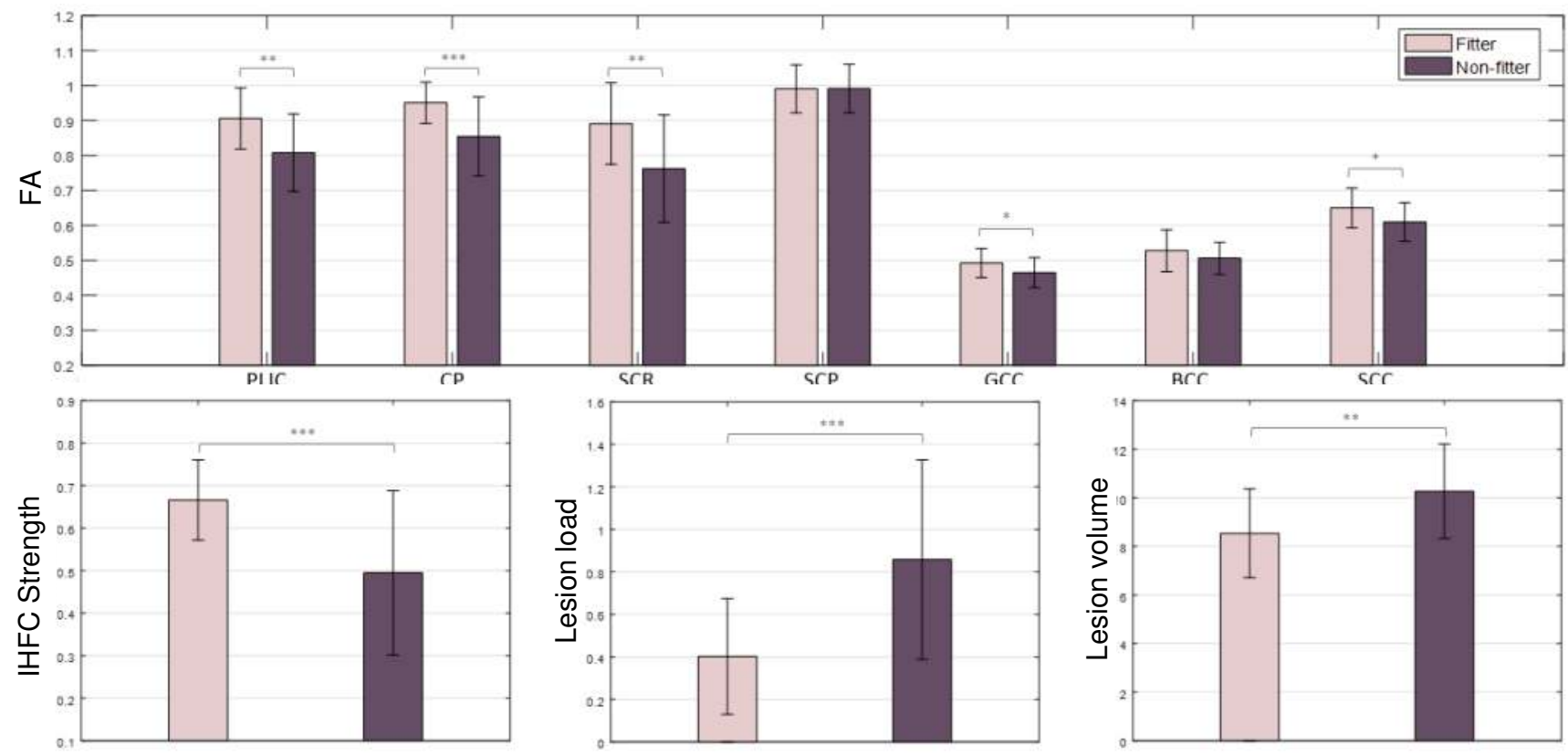

- FA values of the Non-fitters were significantly lower than the Fitters in the PLIC, CP, SCR, GCC, and SCC.

- There were significant differences in the lesion volume and lesion load between the Fitters and Non-fitters. The lesion load of the CST and lesion volume were greater in the Non-fitters than the Fitters.

- In rs-fMRI, the interhemispheric functional connectivity (IHFC) strength in the Non-fitters was lower than the Fitters.

Investigation of Predictive Imaging Markers
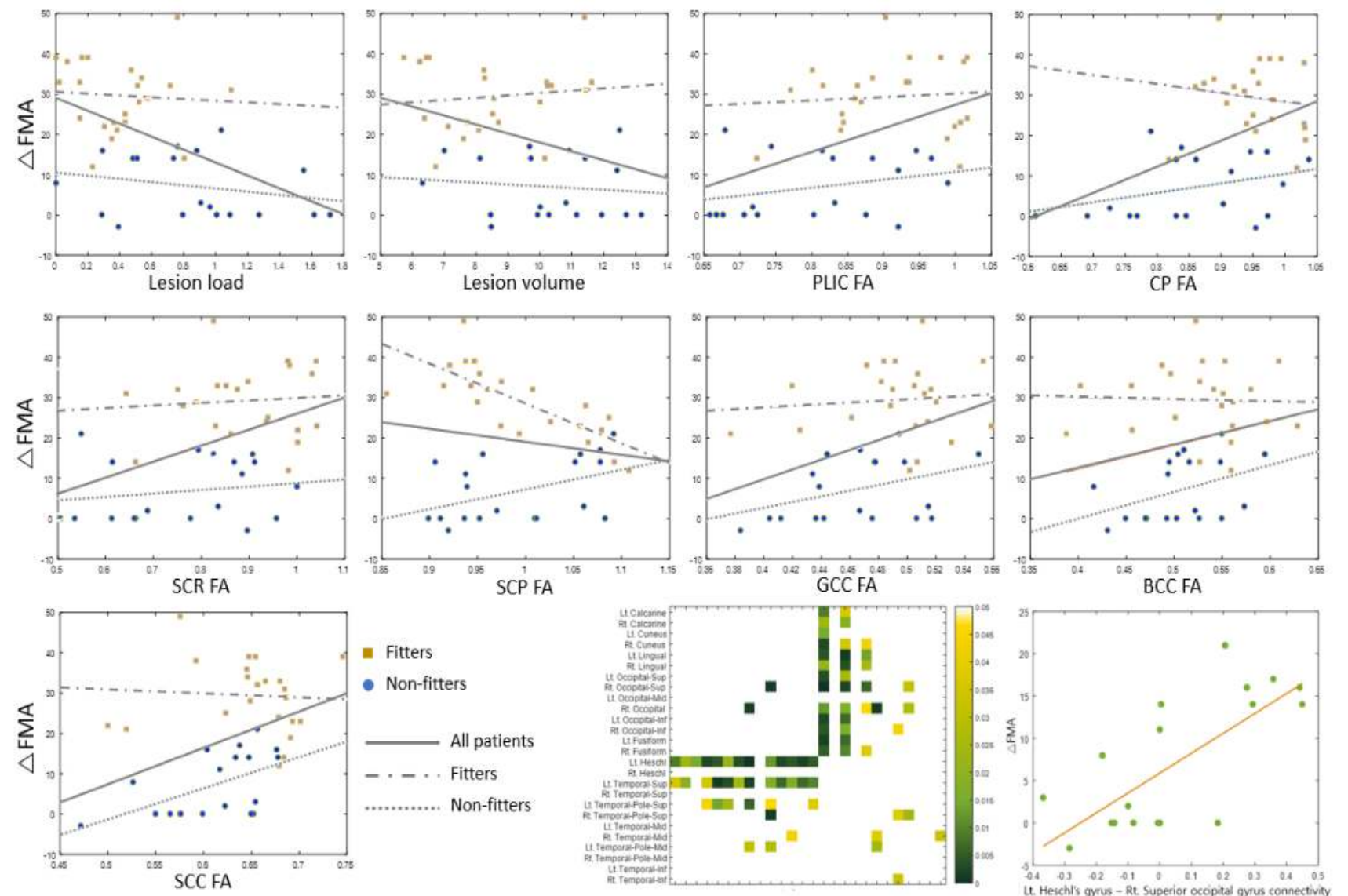

- In the Fitters, the SCP FA value was the significant predictable biomarker $\left(R^{2}=0.569, p<0.001\right)$. In contrast, the SCC FA value was th significant predictable biomarker in the Non-fitters $\left(R^{2}=0.293, p=\right.$ 0.016). Additional analysis of $\mathrm{rs}-\mathrm{fMRI}$ data, the connectivity strength between the left superior temporal cortex and bilateral occipital cortex showed significant correlation with later motor recovery in Non-fitters. The connectivity between the left Heschl's gyrus (HES) and right superior occipital gyrus (SOG) showed the strongest predictive value $\left(R^{2}\right.$ $=0.514, p<0.001)$.

\section{Conclusions}

- The neuroimaging biomarkers for motor recovery were different between both groups. The best predictive marker of each group (SCP FA in the Fitter group; SCC FA and Lt. HES - Rt. SOG FC in the Non-fitter group) showed high prediction power which was higher than PLIC FA.

- This neuroimaging approach is helpful to predict motor recovery in patients with initially severe motor impairment after stroke who are usually considered as highly unpredictable individuals and needed a different strategy for recovery prediction beyond the traditional approaches. 